\title{
EL DISCURSO DE LA MIMESIS EN EL LIBRO X DE LA REPÚBLICA DE PLATÓN: SIMILITUDES Y DIFERENCIAS CON LOS LIBROS II-III
}

Data recepción: 2009/11/02

Data aceptación: 2010/07/10

Contacto autor: jtg.jorge@gmail.com
Jorge Tomás García

Universidad de Murcia

\section{RESUMEN}

El principal objetivo del artículo es señalar las similitudes y diferencias en el tratamiento del concepto de mimesis entre el libro $\mathrm{X}$ de La República y los libros II-III de la misma obra. Dicho análisis resulta fundamental para esclarecer el concepto global de Platón sobre la verdadera función de las artes en su visión política y social del Estado. Para ello, es necesario realizar un análisis detallado del libro X de La República, única obra del corpus platónico en la que trata manera detallada el problema del arte y de la sociedad. Los resultados son sorprendentemente claros. La corrupción y el aspecto peligroso del arte no se atribuye a ningún artista o escuela en concreto, sino que se refiere a la entidad mimética del arte como imitación de una imitación.

Palabras clave: Platón, arte, mimesis, censura, poesía

\section{ABSTRACT}

The main objective of the article is to highlight the similarities and differences in the way in which Book $X$ and Books II and III of The Republic treat the concept of mimesis. This analysis is essential in clarifying Plato's global conception of the true role of the arts in his political and social vision of the State. Such an approach requires a detailed analysis of Book X of The Republic, the only work in which Plato looks at the problem of art and society in depth. The results are surprisingly clear. Rather than attributing corruption and the dangers of art to one artist or school in particular, he refers to the mimetic institution of art as an imitation of an imitation.

Keywords: Plato, art, mimesis, censorship, poetry

En la estructura y contenido del controvertido libro $X$ sorprenden varios temas fundamentales': en primer lugar, el tratamiento general del tema de la $\mu$ í $\mu \eta \sigma \varsigma^{2}$, y en segundo lugar, el tono más agresivo que utiliza en este libro en comparación con los libros II-III. Platón vuelve sobre un tema que ya había tratado con anterioridad, si bien para Jaeger

está absolutamente justificado que Platón examine una vez más, sobre esta base (la base de la teoría de las Ideas), el problema de la poesía ${ }^{3}$.

En el mismo sentido, el repentino cambio del comienzo del libro $X$ se justifica de inmediato, ya que se menciona la división del alma como argumento tratado con anterioridad. De esta mane$\mathrm{ra}$, se puede ahora acometer la lucha contra la poesía, enemiga número uno de la razón, con el sólido argumento del alma ya asentado, con la Teoría de las Ideas expuesta, y con el concepto de "belleza" desarrollado en el Symp. ${ }^{4}$.

El discurso de la imitación en el libro $X$, que Else sitúa unos años después del 360 a.C., ha sido denominado "autocontradictorio" al compararlo con el realizado anteriormente en el libro III . Platón había definido la poesía siguiendo dos parámetros fundamentales: uno, que la poesía se componía en verso ${ }^{6}$, y dos, que sus composiciones eran historias de ficción (PI. Phdn. 69b). La crítica se ha centrado en la comparación del 
trabajo de los poetas con el de los pintores (PI. Rep. 597e-598d); ; se entiende la imitación de ambos artistas como el retrato directo de las apariencias. Así, resulta accesible el concepto de artista literario que tenía Platón; esto es, el del imitador y el que entretiene al público. A la vez, sus obras (los fantasmas de sus recreaciones literarias que resultan ser estímulos directos emocionales) eran interpretadas a la luz de los conocimientos evidentes que posee un imitador $^{8}$, y rechazan todo afán de universalidad ${ }^{9}$ :

porque dicen que es necesario que un buen poeta, si va a componer debidamente lo que compone, componga con conocimiento; de otro modo no será capaz de componer (PI. Rep. 598e).

El imitador es interpretado de manera más global como "el creador de imágenes" (PI. Rep. 601c), afectando a todo tipo de representación o descripción ${ }^{10}$. La literatura se confirma como moralmente inaceptable, y resta tan sólo un camino para poner la irresponsabilidad de la literatura al servicio de los objetivos pedagógicos: subordinarla al Estado, tal y como se encuentra en los libros II-III de Rep. y en II-VII de Lg. ${ }^{11}$, donde el Estado se encuentra capacitado para desarrollar una literatura responsable ${ }^{12}$.

Mientras que en el libro III, en el que Platón estaba realmente interesado en demostrar la verdadera importancia del arte en la vida de la gente, se propone la expulsión de algunos poetas imitativos ${ }^{13}$, únicamente de aquellos que realizaban un uso profundo e indiscriminado de la caracterización dramática, en el libro $X$ todos

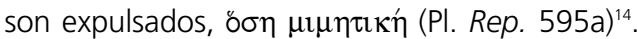
Esta afirmación es el punto principal sobre la que se sustentan los argumentos que señalan a Platón como enemigo irreconciliable de los artistas; pero no debe interpretarse de manera separada o independiente del resto de la obra. La poesía se podía dividir en representativa, sobre todo refiriéndose a la tragedia, y no representativa, considerando ciertas poesías representativas como poco aconsejables para la educación de los jóvenes y de los guardianes, (PI. Rep. 398a).

En esta misma línea, la poesía representativa imitativa no tiene cabida en el Estado debido a su influencia sobre las almas de los espectadores $^{15}$. Especialmente importante es la siguiente afirmación:

al no aceptar de ningún modo la poesía imitativa; en efecto, según me parece, ahora resulta absolutamente claro que no debe ser admitida, visto que hemos discernido las partes del alma (PI. Rep. 595a5).

En este punto se refiere al pasaje en el que se discute la división del alma de los individuos en tres géneros distintos (PI. Rep. 435b). De esta manera, es necesaria la lectura del libro $X$ como coda o remate final a todo el discurso anterior de Rep. para encontrar sentido a las conclusiones finales de Platón ${ }^{16}$. La confirmación de este argumento la encontramos cuando decide conservar ciertas clases de poesía no representativa:

sólo deberán admitirse en nuestro Estado los himnos a los dioses y las alabanzas a los hombres buenos (PI. Rep. 607a).

El ataque de Platón se centra en la poesía representativa imitativa ${ }^{17}$, de diversión, $\dot{\eta} \pi \rho \sigma^{\circ} \sigma$

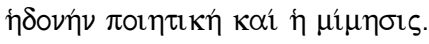

En el libro III (Rep. 397d4-5, 401a), Platón llegó a admitir la posibilidad de una imitación con conocimiento en su Estado, ya que en el libro V (Rep. 475a-480a), había distinguido entre

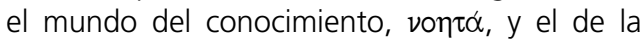
opinión, $\delta \circ \xi \alpha \sigma \tau \alpha$. Tanto el pintor como el poeta llevan a cabo sus actividades artísticas en un nivel inferior al de la verdad, puesto que ontológicamente son tan sólo una copia de la realidad (PI. Rep. 605a9-10). Este argumento es inseparable al del libro VI (Rep. 509-511), en el que establece el símil de "la línea dividida" a partir del concepto de cik $\alpha \sigma i \alpha$, , conjetura" o incluso "imagen". Algunos de los errores más usuales de la audiencia griega en relación con la poesía y las demás artes miméticas preocupaban especialmente a Platón ${ }^{18}$. Destaca aquel según el cual los jóvenes pensaban que las obras del pintor, al ser vistas desde la distancia, pasaban por ser reales (PI. Rep. 598c1-4). La audiencia tenía en muy alta estima creativa al artista imitador, conocedor de todos los oficios (PI. Rep. 598c6); tam- 
bién piensan que Homero conoce todas las artes, en relación con la excelencia, el malogro y los asuntos divinos, (PI. Rep. 598d7-e2) ${ }^{19}$; de igual manera, creen que el trabajo de los imitadores es la verdad y no la apariencia, (PI. Rep. 598e5 - 599a3); por lo tanto, los buenos poetas conocen realmente lo adecuado y que la mayoría considera oportuno (PI. Rep. 599a4). El imitador imitará lo que parezca bello a la multitud ignorante, aunque no sepa si cada cosa es buena o mala (PI. Rep. 602b1-4), y los artistas engañan a la audiencia porque realizan su obra lejos de la verdad, y se dirigen a la parte de nuestra alma que está lejos de la sabiduría, (PI. Rep. 603a8); este proceso creativo es contrario a la razón y a la ley, (PI. Rep. 604a10).

Los últimos puntos citados están referidos a una nueva división del alma planteada en Rep. 602e-603a, según la cual existe una parte dedicada y preparada para la medición y otra para la opinión, y la misma parte del alma no puede emitir opiniones contrarias sobre lo mismo. Con anterioridad había definido el alma como

una única figura de una bestia polícroma y policéfala, que posea tanto cabezas de animales mansos como de animales feroces, distribuidas en círculo, y que sea capaz de transformarse y de hacer surgir de sí misma todas ellas (PI. Rep. 588c).

Esta lectura es consecuencia del orden moral jerárquico establecido por Platón con la razón como la dominadora de la fuerza y de los apetitos $(\text { Rep. 605a-b) })^{20}$. A continuación, vuelve a insistir sobre el mismo punto:

la parte del alma que estaba hambrienta de lágrimas y quejidos y buscaba satisfacerse adecuadamente... ésa es la parte a la que los poetas satisfacen y deleitan: en tanto que lo que es por naturaleza lo mejor de nosotros, dado que no ha sido suficientemente educado ni por la razón ni por la costumbre, afloja la vigilancia de la parte quejumbrosa, en cuanto que lo que contempla son aflicciones ajenas... (PI. Rep. 606a3-b5) 21.

El comienzo del libro X de Rep. debe ser leído e interpretado como complemento y continua- ción del libro III, ya que en dicho libro habla de anteriores conclusiones y, al afirmar que deben admitirse himnos a los dioses y panegíricos a los hombres buenos, no da más explicaciones, como si todo hubiese sido tratado con anterioridad en el libro III2. Dicho punto continúa con lo afirmado en Rep. 397d4-5: "si mi opinión se impone, admitiremos la imitación pura del hombre de bien", obligando a desterrar de manera definitiva la idea implantada erróneamente que nos hace creer que Platón quería dejar a su Estado Ideal absolutamente desposeído de las manifestaciones artísticas. Así, es necesario realizar una doble lectura de la $\mu$ í $\mu \eta{ }^{\prime} \varsigma$ en Platón ${ }^{23}$ : un primer tipo, meramente imitativo ya que imita las apariencias de las cosas apreciables por los sentidos (PI. Rep. 598b), y en la que el poeta trágico y el pintor representan la apariencia de los objetos sin preocuparse de su auténtica naturaleza; y el segundo tipo, que es la imitación del mundo de las Ideas, la cual sólo puede ser conseguida por el individuo con conocimiento (PI. Rep. 402b-c,500d), y si los poetas aceptan las censuras filosóficas (PI. Lg. 719b), pueden llegar a convertirse en filósofos.

Esta diferencia entre la imitación utilizada en el libro III y la del libro $X$ es insuficiente para resolver el aparente conflicto entre ambos libros 24 ; pues, mientras en el libro III se permiten el género del ditirambo y poemas en los que los hombres honorables son enaltecidos, en el libro $X$ se excluye, aparentemente, a todo tipo de artista imitativo. Así, la función del arte es la de impartir conocimientos, pero como se muestra incapaz de hacerlo, debe introducir a los jóvenes en la armonía del carácter y familiarizarlo con las rectas opiniones ${ }^{25}$. Pero lo que resulta evidente es que $\mu$ í $\mu$ ᄁors se refiere ahora al acto poético en su totalidad y no tan sólo a una versión dramáti$\mathrm{ca}^{26}$. Para Platón no toda imitación es arte, aunque la imitación es una condición indispensable para que algo sea arte.

La amplitud de significados que puede llegar a tener al término $\mu$ í $\mu \eta$ ors en el conjunto de la cultura griega arcaica se unifican al llegar a

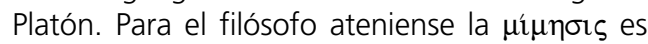
el instrumento creativo que utiliza el artista imitativo, y el uso de este instrumento puede llegar a resultar dañino para la sociedad. La creación de imágenes, عı̌ $\delta \omega \lambda$ ov, es dividida en dos clases; 


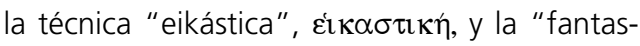
magórica", (PI. Sph. 235a10 - 236d8), y se rea-

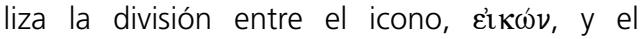
fantasma, $\phi \alpha \dot{v} \tau \alpha \sigma \mu \alpha$. El artista imitativo es semejante al sofista, ya que es capaz de domi-

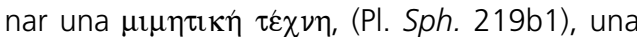
"técnica mimética", que emplea con un determinado fin totalmente opuesto al requerido por Platón, y que se muestra incapaz de revelar la esencia de la técnica. Ambos son creadores de $\phi \alpha \nu \tau \alpha ́ \sigma \mu \alpha \tau \tau$, , "fantasmas", que nos alejan de la auténtica y única realidad ${ }^{27}$.

Por lo tanto, los artistas imitativos, si bien pueden y son capaces de desempeñar un importante papel educativo dentro de la educación de los guardianes, tal y como se demuestra en los libros II y III, normalmente optan por la peor de las imitaciones, la que es citada al comienzo del libro X de la Rep. Por lo tanto, la $\mu$ í $\mu \eta \sigma r s$ se debe encuadrar en el proyecto global educativo y social que llevaba a cabo Platón, y la pregunta debería ser la siguiente, ¿qué función tiene el arte, siguiendo a Heidegger, en ese proceso de "conversión del alma entera", $\pi \alpha_{1} \delta \varepsilon i \alpha$, para poder llegar al "desencubrimiento", $\dot{\alpha} \lambda \dot{\eta} \theta \varepsilon\llcorner\alpha$, de la esencia de las cosas en la sociedad ideal planteada por Platón? El significado de $\dot{\alpha} \lambda \dot{\eta} \theta \varepsilon \dot{\varepsilon} \alpha$ es opuesto al

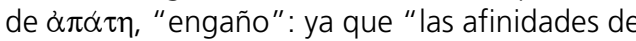
la $\delta \sigma \xi \alpha$ con la $\alpha \pi \alpha \dot{\tau} \eta$ y las formas de la ambigüedad pueden encontrar su confirmación en determinadas significaciones fundamentales de la $\delta 6 \xi \alpha^{\prime \prime 28}$. Lo que Platón buscaba era el Estado Ideal, que casi llegaba a ser una antiAtenas del siglo V-IV a.C.; una u-topía y una eutopía, un no-lugar y un buen-lugar, que como toda utopía se inscribe en un guión destinado a su propio cumplimiento ${ }^{29}$.

\section{BIBLIOGRAFIA}

Babut, D. (1985): "Sur la notion d'Imitation dans les doctrines esthétiques de la Gréce classique" REG 98 ,72-92.

(1985): "Paradoxes et énigmes dans l'argumentation de Platon au livre $X$ de la République" Historie et Structure (Hommages á Victor Goldschmidt) Paris.

Baeumler, A. (1934): Ästhetik, Berlin.

Belfiore,E.(1983): "Plato's Greatest Accusation against Poetry" CJPh 9, 39-62.

Collingwood, R.G. (1925): "Plato's Philosophy of Art" Mind 34, 154-172.

Corlett, J.A. (1997): "Interpreting Plato's Dialogues" CQ N.S. 47:2, $423-$ 437.
Detienne, M. (1982): Los maestros de la verdad en la Grecia arcaica, Madrid.

Dubois, P. (2006): "The History of the Impossible: Ancient Utopia" CIPh 101:1, 1-15.

Dyson, M. (1988): "Poetic Imitation in Plato Republic 3" Antichton 22 ,42-53.

Else, G.F. (1972): The Structure and the Date of Book 10 of Plato's Republic, Heidelberg.

Gilbert, A.H. (1939): "Did Plato Banish the Poets or the Critics?" StudPhilol. $36,1-19$.

Goodrich, R.A. (1982): "Plato on Poetry and Painting" BritJAesthet. 22:2, 126-137.

Griswold, C. (1981): "The Ideas and the Criticism of the Poetry in Plato's Republic, Book 10" JHistPhilos. $19: 2,135-150$
Gulley, N. (1954): "Plato's Theory of Recollection" CQ 4:3/4, 194-213.

(1977): "Plato on Poetry" G\&R 24:2, 154-169.

Hall, R.W. (1974): "Plato's Theory of Art: A Reassessment" JAesthetArtCritic. 33:1, 75-82.

Halliwell, S. (2002): The Aesthetics of Mimesis. Ancient texts and Modern Problems, Princeton.

Havelock, E. A. (1982): Preface to Plato, Harvard.

Huber-Abrahamowicz,E. (1954) Das Problem der Kunst bei Platon, Winterthur.

Jaeger, W. (1957): Paideia, Méxi$\mathrm{co}$.

Janaway, C. (1991): "Plato's Analogy Between Painter and Poet" BritJAesthet. 31:1, 1-12.

Mastrangelo, M. - Harris, J. (1997): "The Meaning of Republic 
606a3-b5" CQ 47:1,301-305.

Murphy, N.R. (1951): The Interpretation of Plato's Republic, Oxford.

Murray, P. (1996): Plato on Poetry. Ion; Republic 376e-398b9; Republic 595-608b10, Cambridge.

Nehamas, A. (1988): "Plato on Imitation and Poetry in Republic 10" Plato. Critical Assesments, Smith, N.D. (ed.), Volumen III, Routledge ,296323.

Oates, W.J. (1941): "Plato's Philosophical Creative Artist" CW 35, 247.

Philip, J.A. (1961): "Mimesis in the Sophistes of Plato" TAMPhilolAssoc 92 , 453-468.
Rucker, D. (1966): "Plato and the Poets" JAesthetArtCritic. 25:2, 167170.

Rutenber, C.G. (1946): The Doctrine of Imitation of God in Plato, Nueva York.

Tate, J. (1928): "Imitation in Plato's Republic" CQ 22:1,16-23.

(1929): "Plato and Allegorical Interpretation" CQ 23, 142-54.

(1930): "Plato and Alegorical Interpretation(continued)" CQ 24,110.

(1932): "Plato and Imitation" CQ 26:3/4, 161-169.

(1932): "Plato and Imitation" CQ
26,161-69.

(1932): "Plato Republic 598 E" CIPh 27:3, 282-284.

Schipper,E.W. (1963): "Mimesis in the Arts in Plato's Laws" JAesthetArtCritic. 22:2,199-202.

Shorey, P. (1932): "Note on Plato Republic 598E" CPh 27:1, 85

Slings, S.R. (2003): "Critical Notes on Plato's Politeita, IX" Mnemosyne 56:4, 449-463.

Walter, J. (1967): Die Geschichte der Ästhetik im Alterum, Hildesheim.

White, N.P. (1979): A companion to Plato's Republic, Oxford.

\section{NOTAS}

${ }^{1}$ Según White, N.P. (1979) dentro de Rep. se encuentran en ocasiones digresiones que no siguen un orden natural, encontrando el máximo ejemplo en 608b-608c cuando pasa repentinamente de hablar sobre la poesía a tratar directamente el tema de la inmortalidad del alma y sus respectivas consecuencias.

2 Babut, D. (1985) 133.

${ }^{3}$ Jaeger, W. (1957) 764-765.

${ }^{4}$ Huber-Abrahamowicz, E. (1954) 9-14: analiza el significado filosófico del concepto de belleza relacionando la visión del Symp. con algunos fragmentos del Tim. 29a, 30a, en el segundo capítulo "Die philosophische Bedeutung des Schönen"; Walter, J. (1967) 202, "Kosmetische Elemente des Schönen"; Baeumler, A. (1934) 317, "Die Idee des Schönen".

5 Tal y como apunta Else,

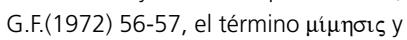
similares van sustituyendo progresivamente a $\mu \dot{\varepsilon} \theta \varepsilon \xi ı$, "participación", para representar la relación entre los particulares y las Formas. También en Rutenber, C.G. (1946) 21, para la rela-

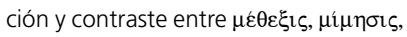

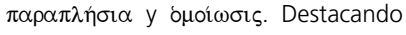

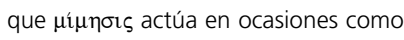
metáfora en la obra de Platón.

${ }^{6}$ Grg. 502c, Symp. 205c, Rep. 393d, 601d, 607d, Phdr. 258d.

${ }^{7}$ Janaway, C. (1991) 1-12; Rucker, D. (1966) 167-170; Goodrich, R.A. (1982) 126-137.

${ }^{8}$ Gulley, N. (1977) 154-169.

${ }^{9}$ Shorey, P. (1932) 85.

${ }^{10}$ Murphy, N.R. (1951) 224.

${ }^{11}$ Schipper,E.W. (1963) 199-202. Las artes miméticas en $L g$., entendiendo por "artes miméticas" el conjunto de música, danza, poesía, drama, pintura y escultura, pueden tener formas maravillosas y no sólo centrarse en la representación de las apariencias.

12 Para una justa interpretación de la relación entre los distintos diálogos platónicos Corlett, J.A. (1997) 423-437.

${ }^{13}$ Dyson, M. (1988) 42-53.

${ }^{14}$ El prefijo -ıк乏 al final de una palabra en griego señala arte o ciencia, mientras que -ıќ́s se refiere al experto en realizar dicho arte o ciencia. Gilbert, A.H.(1939) 1-19.

15 Como Collingwood, R.G. (1925) 151.
${ }^{16}$ Especialmente del libro IX, ver Slings, S.R. (2003) 449-463.

${ }^{17}$ Nehamas, A. (1998) 296-323; Halliwell, S. (2002) 150.

${ }^{18}$ Belfiore, E. (1983) 39-62.

${ }^{19}$ Griswold,C.(1981)135-150: en el libro X Rep. cita a Homero y Hesíodo pero no nombra sus obras, en los libros II-III de la Rep. ataca más a la llíada que a la Odisea (libro II: 7 veces llíada- 2 Odisea, libro III: 25 Ilíada- 8 Odisea).

${ }^{20}$ Hall, R.W. (1974) 75-82.

${ }^{21}$ Una interpretación filológica de este fragmento se encuentra en Mastrangelo, M.- Harris, J. (1997) 301-305.

22 Tate, J. (1928) 16-23; (1932) 161-69; (1932) 282-284; (1929) 14254; (1930) 1-10.

${ }^{23}$ Oates, W.J. (1941) 247; Babut, D. (1985) 72-92.

${ }^{24}$ Murray, P. (1996) 228-229.

${ }^{25}$ Tate, J. (1932) 161-169.

${ }^{26}$ Havelock, E. A. (1982) 26.

${ }^{27}$ Para el significado y el uso de $\mu$ í $\mu$ $\sigma \varsigma s$ en el Sph., ver Philip, J.A. (1961) 453-468; Gulley, N. (1954) 194-213.

${ }^{28}$ Detienne, M. (1982) 116.

${ }^{29}$ Dubois, P. (2006) 1-15. 